\title{
ANALYSIS OF DYNAMIC CHANGEOF HONG JIANNAO LAKE BASED ON SCALED SOIL MOISTURE MONITORING INDEX
}

\author{
H. Yue ${ }^{1}, Y$. Liu $^{1 *}$ \\ ${ }^{1}$ College of Geomatics, Xi'an University of Science and Technology, Xi'an, Shaanxi 710054, China - yue_hui@live.com, \\ liuying712100@163.com
}

Commission III, WG III/9

KEY WORDS: Scaled Soil Moisture Monitoring Index, Hongjiannao Lake, Dynamic Change

\begin{abstract}
:
Land surface water plays an important role in ecological balance and human survival. It is experiencing some dramatic changes due to climate change and human activities, Hong Jiannao Lake located in the arid and semi-arid area of China, it played a very important role in the regulation of the local climate, the balance of water resources and the maintenance of biological diversity. Hongjiannao Lake area in recent years continues to shrink, it was urgent to get the Hongjiannao Lake area change trend. This article take Hongiiannao Lake as study object using MODIS image of NIR and Red wavelength reflectivity data, selected April to October of 2000-2014,consturcted scale of SMMI (S-SMMI) based on soil moisture monitoring index (SMMI). The result indicated that lake area reduced from $46.9 \mathrm{~km}^{2}$ in 2000 to $27.8 \mathrm{~km}^{2}$ in 2014 , average decay rate is $1.3 \mathrm{~km}^{2} / \mathrm{a}$. The lake's annual change showed a trend of periodic change. In general, the lake area began to increase slowly each year in April, and the area of the lake area reached the maximum, and then decreased gradually in June to July. Finally, we analysed the main driving factors included natural, man-made, and underground mining which lead to the lake area shrink.
\end{abstract}

\section{INTRODUCTION}

Lake water in arid and semi-arid area is a vital recourse and plays an important role in ecological balance. It is experiencing some dramatic changes due to climate change and human activities (Ma, 2010). Therefore, it is necessary to extract water bodies from non-water features and evaluate the spatiotemporal distributions and changes of land surface water. Remote sensing technique is an effective approach in monitoring water resources because of providing large-scale, long-term, repeated and real-time information (Sun et al., 2012; Verpoorter et al., 2012). Based on multi-resource remotesensing data and many water body-extracting methods, numerous studies have focused on delineating the boundaries and dynamic changes of land surface water bodies. The multiband spectral water index method is among the most widely used approach in distinguish water features and basically referred to blue, green, near-infrared, middle infrared and shortwave infrared bands but not involved in red band ( $\mathrm{Li}, 2013$ ). For instance, Feng et al. (2012) used a moderate resolution imaging spectro-radiometer (MODIS) medium resolution (250m) data which collected data from 2000 to 2010 and the Floating Algae Index to assess the short- and long-term characteristics of Poyang lake inundation. McFeeters et al. (2013) noted that high resolution QuickBird imagery combined with normalized difference water index (NDWI) can extract swimming pools for mosquito abatement. Xu (2006) found that the NDWI of McFeeters (2013) fails to suppress the built-up land noise, replace the NIR band with the middle infrared (MIR) band, and then establish the modified NDWI (MNDWI). The open water features can be enhanced using this index, and the built-up land noise, as well as vegetation and soil noise, can be suppressed and even removed efficiently (Liu 2015). The two methods NDWI and MNDWI are widely used for land surface water extraction, land surface water mapping, mosquito habitat recognition, ecological assessment, and land cover change. In this paper, we constructed a water extraction index, i.e. scaled soil moisture monitoring index (SSMMI) in NIR-Red spectral space.

\section{METHODS}

In this paper, we constructed a water extraction index, i.e. scaled soil moisture monitoring index (SSMMI) in NIR-Red spectral space. The reflectance of water in red and NIR bands is lower than vegetation, bare soil and built-up land (Figure 1). Therefore, the distance of water pixels to the origin $\mathrm{O}$ is less than the distance between other land cover type pixels and the origin $\mathrm{O}$. We can use the distance between any point $\mathrm{E}$ and the origin $\mathrm{O}$ to recognize water pixels from non-water pixels, i.e. objects placed near to the point $\mathrm{O}$ are always water bodies or extremely wet regions (Figure 2). The threshold values of NDWI and MNDWI are zero and are reasonable starting thresholds of AWEI. In this paper, dynamic threshold values were used to delineate the water bodies of three test sites. The Otsu method (maximum between-class variance method) is a dynamic method that was applied to acquire the threshold for separating water bodies from the non-water features.

In this paper, by using remote sensing image on the phase is not completely consistent, to eliminate the phase difference, we proposed the calculation method of SSMMI scale, which can be defined as:

$$
S-S M M I=\frac{S M M I-S M M I_{0}}{S M M I_{S}-S M M I_{0}}
$$

* Corresponding author 
Where SMMI refers to a certain like SMMI value of one pixel; SMMI0 refers to the saturation of the bare earth SMMI value; SMMIs refers to the dry area of bare soil SMMI values. In this case, in combination with the practical situation of the study area, SMMI0 values to select each image SMMI cumulative frequency stated confidence level of $2 \%$ of SMMI value, SMMIs value selection SMMI cumulative frequency stated confidence level of $98 \%$ of SMMI values. In the process of calculation, the value of S-SMMI has three parts.

$$
S-S M M I= \begin{cases}0 & S M M I<S M M I_{0} \\ \frac{S M M I-S M M I_{0}}{S M M I_{S}-S M M I_{0}} & S M M I_{0}<S M M I<S M M I_{S} \\ 1 & S M M I>S M M I\end{cases}
$$

Using the formula (2) S-SMMI image in the study are can be calculated, and where the S-SMMI value was 0 can be defined as water-body.
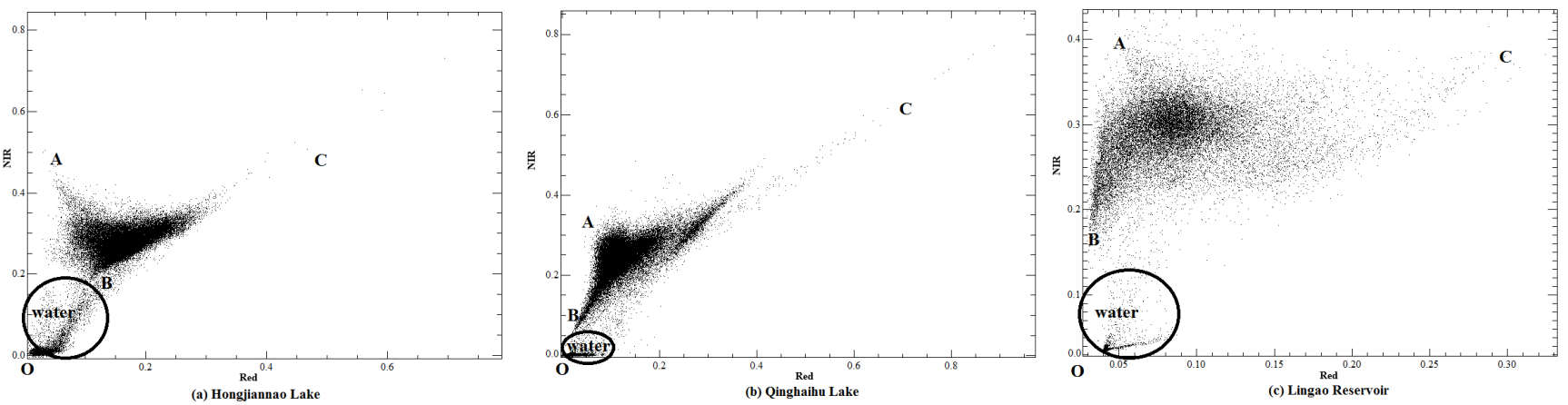

Figure 1. Spectral space using reflectance data of red and NIR bands

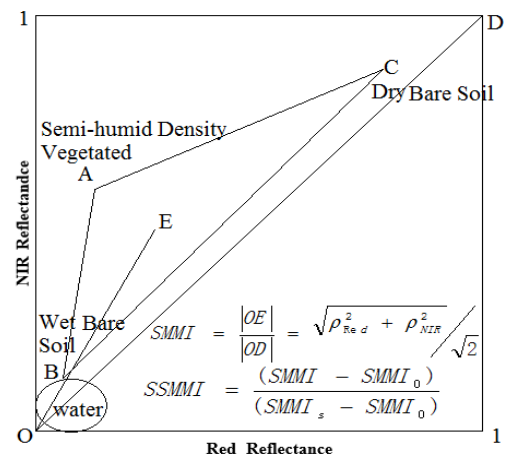

Figure 2. Sketch map of Scaled Soil Moisture Monitoring Index (SSMMI)

\section{STUDY AREA AND DATA ACQUISITION}

\subsection{Study Area}

Hong Jiannao Lake is the largest desert freshwater lake in semiarid and arid region of China, which played an important role in adjusting the local climate, water resource balance and biological diversity. It located at the border of Shaanxi Province and Inner Mongolia Autonomous Region, centered at $\left(110^{\circ}\right.$ $52^{\prime} 30^{\prime \prime}$ E, $\left.39^{\circ} 06^{\prime} \mathrm{N}\right)$. The position in the south-west of Shendong mining area, which was one of China's biggest coalproducing regions (Figure 3). The lake-water area was 61.93 $\mathrm{km}^{2}$ in 1978. However, some studies found that the area of Hong Jiannao Lake decreased rapidly in the past decades, influenced the ecological and economic functions of Hong Jiannao Lake, due to climate change and human activities, especially the underground mining activities (Liu, 2015). Therefore, it is necessary to obtain the accuracy area and monitoring the dynamic change of Hong Jiannao Lake. Then, analysis the law between lake area change and underground mining.

\subsection{Data Acquisition}

In NASA's web site (http://revert. echo.nnsn.gov/revert/) download MODIS MOD13Q1 data which year from 2000 to 2014 on April to October, the resolution of the remote sensing images is $250 \mathrm{~m}$. MOD13Q1 subject products belong to product of MODIS data of land, the full title called the MODIS/Terra Vegetation Indices 16-Day L3 SIN Global $250 \mathrm{~m}$ Grid, which is a level 3 Grid land Vegetation data products, it adopts the Sinusoidal projection method, the spatial resolution of $250 \mathrm{~m}$, provides a data every 16 days, is large scale high phase data.

\section{ACCURACY ASSESSMENT}

Using S-SMMI formula 1 to extract the Hong Jiannao Lake borders and use the measured data for validation which actual measurement in August 2004. Accuracy calculation formula can be defined as

Error $_{i}=\frac{W A_{\mathrm{ex}}-W A_{\mathrm{tr}}}{W A_{\mathrm{tr}}} \times 100 \%$

Where Errori refers to S-SMMI extract water body boundary error, WAex refers to the water body which calculated by SSMMI, WAtr refers to the true water body. 


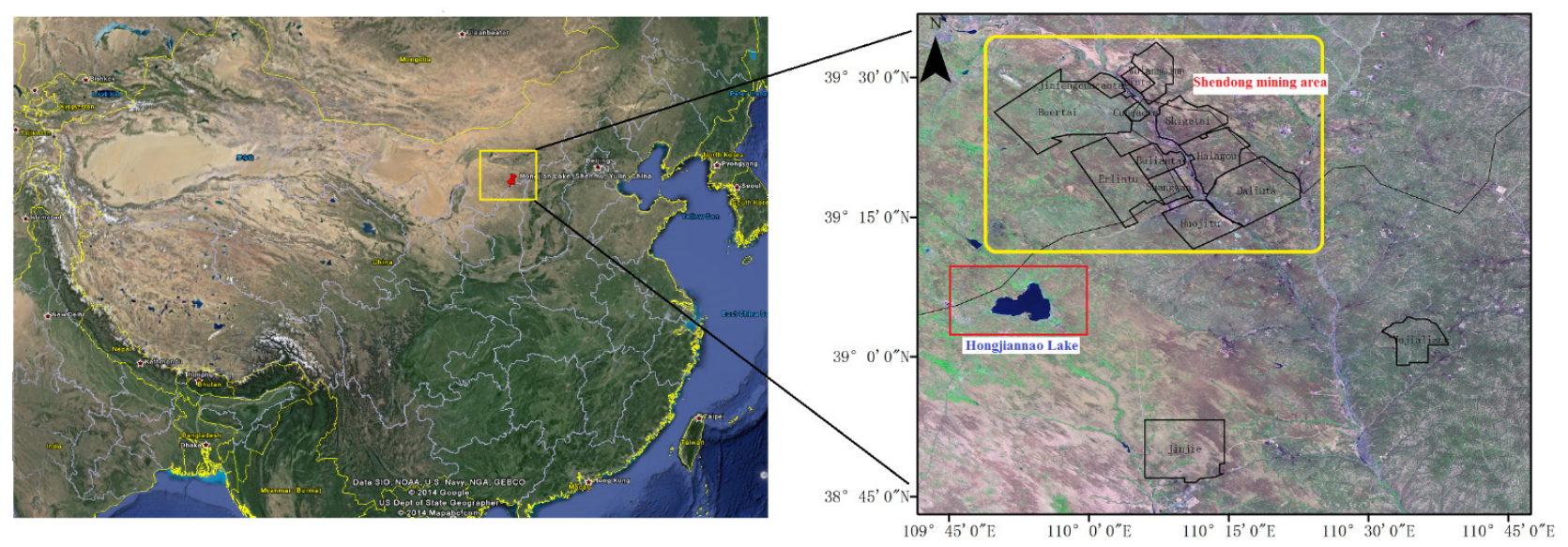

Figure 3. Location of Hongjiannao Lake

\section{RESULTS AND DISCUSSION}

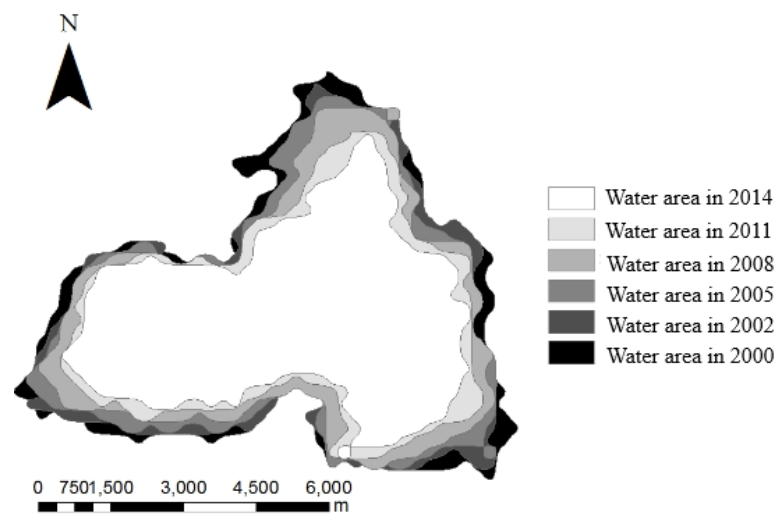

Figure 4. The schematic diagram of area reduced of Hong Jiannao Lake

Because of the lack of remote sensing image data of the Hong Jiannao Lake before 2000, so in this study the scope of the Hong Jiannao Lake area which focused on the year between 2000 and 2014. Extraction water bodies using remote sensing image data out of the lake area in 2000-2014, the data. The Hong Jiannao Lake area change tendency has a certain regularity within a year. Overall, lakes area increased slowly, when started in April every year to July-August where the lake area reached the largest point, and then decreased. When compared with the actual measurement in August 2004, the Errori was $3.95 \%$, the results obtained small errors. According to the literature, Hong Jiannao Lake was formed in the 1920s, the idea was consistent with the result which obtained from the recent use of the Hong Jiannao Lake bed sediment in the performance of the history of the formation and development of the Hong Jiannao Lake. After that the lake area increased slowly, in the 35 years from 1956 to 1991, Hong Jiannao Lake area expanded $26.03 \mathrm{~km}^{2}$, lake area is continuously decreasing after 1991. After 2000, using the remote sensing image data to extract the inter-annual variability of the lake area in 2000-2014, which showed that the area of the lakes in the study area are still present a shrinking trend, lake covers an area of $46.9 \mathrm{~km}^{2}$ decreased from 2000 to 2000 years of $27.8 \mathrm{~km}^{2}$, change rate of $41 \%$, annual average attenuation rate is $1.3 \mathrm{~km}^{2}$. In the year 2004-2005, the rate of change of the smallest, only $0.48 \%$, and the rate of change of the maximum in $2010-2011$ at $8.4 \%$, the rate of change of the rest of the years in between $1.7 \%$ and $4.7 \%$.

In order to more intuitive to express the change of lake area, it is selected the 2000, 2002, 2005, 2008, 2011 and 2014 of Hong Jiannao Lake after image processing, add up, the result is showed in Figure 4. Many scholars analyzed the reasons of Hongjiannao Lake area decreased, the majority of opinions thought that there were several factors contributing to the shrinking Lake area, and general influence factors on precipitation, drought and other natural factors as well as agricultural irrigation, reservoirs, blocking, consider factors such as underground coal mining.

\section{ACKNOWLEDGEMENTS (OPTIONAL)}

This work is partially supported by grants from The National Natural Science Foundation of China (Grant No. 41401496).

\section{REFERENCES}

Feng, L.; Hu, C.; Chen, X.L.; Cai, X.B.; Tian, L.Q.; Gan, W.X.,2012. Assessment of inundation changes of Poyang Lake using MODIS observations between 2000 and 2010. Remote Sensing of Environment, 121(2), pp.80-92.

Li, W.B.; Du, Z.Q.; Ling, F; Zhou, D.D.; Wang, H.L.; Gui, Y.M.; Sun, B.Y.; Zhang, X.M.,2013. A Comparison of Land Surface Water Mapping Using the Normalized Difference Water Index from TM, ETM+ and ALI. Remote Sensing, 5(11), pp.5530-5549.

Liu Ying, Wu Lixin, Yue Hui., 2015. Bi-parabolic NDVI-Ts Space and Soil Moisture Remote Sensing in an Arid and Semiarid Area. Canadian Journal of Remote Sensing, 41(3), pp.159-169.

Ma, R. H.; Duan, H.T.; Hu, C.M.; Feng, X.Z.; Li, A.N.; Ju, W.M.; Jiang, J.H.; Yang, G.S., 2010. A half-century of changes in China's lakes: Global warming or human influence. Geophysical Research Letters, 2010, 37, pp.106-108.

McFeeters, S.K., 2013. Using the Normalized Difference Water Index (NDWI) within a Geographic Information System to Detect Swimming Pools for Mosquito Abatement: A Practical Approach. Remote Sensing, 5(7), pp.3544-3561.

Sun, F.; Sun, W.; Chen, J.; Gong, P., 2012. Comparison and improvement of methods for identifying water bodies in 
remotely sensed imagery. International Journal of Remote Sensing, 33(21), pp.6854-6875.

Verpoorter, C.; Kutser, T.; Tranvik, L., 2012. Automated mapping of water bodies using Landsat multispectral data. Limnology \& Oceanography Methods, 10(12), pp.1037-1050.

Xu. H.Q., 2006. Modification of normalised difference water index (NDWI) to enhance open water features in remotely sensed imagery. International Journal of Remote Sensing, 27(14), pp.3025-3033. 